\title{
3 Research Square

\section{Pathogenic Variants Identified using Whole-exome Sequencing in Chinese Patients with Primary Ciliary Dyskinesia}

\section{Yutian Ye}

shenzhen people's hospital

\section{Qijun Huang}

shenzhen people's hospital

Lipeng Chen

shenzhen people's hospital

Chunxian Liang

shenzhen people's hospital

Kaixue Zhuang

shenzhen people's hospital

Tao Liu

shenzhen people's hospital

Fang Yuan

shenzhen people's hospital

Xiangxia Zhang

shenzhen people's hospital

Jie Li

shenzhen people's hospital

Rongchang Chen

shenzhen people's hospital

Yingyun Fu

shenzhen people's hospital

Yongjian Yue ( $\nabla$ yueyongj@163.com )

shenzhen people's hospital

\section{Research Article}

Keywords: primary ciliary dyskinesia, DNAAF3, DNAl1, whole-exome sequencing

Posted Date: February 9th, 2021

DOI: https://doi.org/10.21203/rs.3.rs-156279/v1 
License: (c) (i) This work is licensed under a Creative Commons Attribution 4.0 International License. Read Full License 


\section{Pathogenic variants identified using whole-exome sequencing in Chinese patients with primary ciliary dyskinesia}

1 Yutian Ye ${ }^{1 *}$, Qijun Huang ${ }^{*}$, Lipeng Chen ${ }^{*}$, Chunxian Liang ${ }^{1}$, Kaixue Zhuang ${ }^{1}$, Tao Liu',

2 Fang Yuan ${ }^{1}$, Xiangxia Zhang ${ }^{1}$, Jie Li $^{1}$, Rongchang Chen ${ }^{1,2}$, Yingyun Fu ${ }^{1 \#}$, Yongjian Yue ${ }^{1 \#}$

$3{ }^{1}$ Key Laboratory of Shenzhen Respiratory Diseases, Department of Pulmonary and Critical Care

4 Medicine, Shenzhen Institute of Respiratory Disease, The First Affiliated Hospital of Southern

5 University of Science and Technology, The Second Clinical Medical College of Jinan University,

6 Shenzhen People's Hospital, Shenzhen, Guangdong, China;

$7 \quad{ }^{2}$ State Key Laboratory of Respiration Diseases, the first affiliated hospital of Guangzhou medical

8 University, Guangzhou, Guangdong, China;

$9 \quad$ \# Yutian Ye, Qijun Huang and Lipeng Chen contributed equally to this work.

10

11 * Correspondence: Yongjian Yue

12 Address: Shenzhen People's Hospital, No. 1017 Dongmen North Road, Luohu District, Shenzhen

13 518020, China.

14 Email: yueyongj@163.com

15 Tel: +8615919909356 

518020, China.

Tel: +86 13602582239

21 Fax: +86 0755-22943121

22

23

24

25

26

27

28

29

30

31

32

33

34

35

36

37 


\section{Pathogenic variants identified using whole-exome sequencing in}

Chinese patients with primary ciliary dyskinesia

\section{Abstract}

Background: Primary ciliary dyskinesia (PCD) is an autosomal recessive disorder. The genetic factors contributing to PCD pathogenesis remain elusive for approximately $20-35 \%$ of patients with complex and abnormal clinical phenotypes. Our study aimed to identify causative variants of sporadic PCD genes using whole-exome sequencing (WES).

Result: All patients were diagnosed with PCD based on clinical phenotype or transmission electron microscopy (TEM) images of cilia. WES and bioinformatic analysis were then conducted for patients with PCD. Identified candidate variants were validated by Sanger sequencing. Pathogenicity of candidate variants was then evaluated using in silico software and the American College of Medical Genetics and Genomics (ACMG) database. In total, 15 rare variants were identified in five patients with PCD. Five new variants of $C C D C 40, D N A H 1, D N A A F 3$, and DNAII were considered causative variants and included one splicing and three homozygous variants.

Conclusion: Our study demonstrated that patients with PCD carry rare causative variants of multiple genes. Our findings indicated that not only known causative genes but also other functional genes should be considered for heterogeneous genetic disorders. 
Keywords: primary ciliary dyskinesia, $D N A A F 3, D N A I 1$, whole-exome sequencing

\section{Backgroud}

Primary ciliary dyskinesia (PCD) is an autosomal recessive disorder characterized by respiratory distress, tympanitis, sinusitis, and bronchiectasis [1]. Kartagener syndrome (MIM\# 244400) is a subtype of PCD exhibiting the situs inversus phenotype. The estimated prevalence of PCD ranges from 1 in 10,000 to 1 in 15,000 among Europeans [2]. PCD is diagnosed by measuring nasal nitric oxide and through brush biopsy [3]. Transmission electron microscopy (TEM) is used to obtain a definitive diagnosis by visualizing the ultrastructure of cilia. Male PCD patients present with infertility due to dysfunctional immotile or dyskinetic sperm flagella [4].

Genetic screening has improved our understanding of the pathogenesis of inherited PCD [5]. Previous genetic and functional studies revealed a series of PCD-associated genes causing congenital ultrastructural abnormalities resulting in dysfunctional immotile cilia [6]. Inherited genetic variations are present in approximately $60 \%$ of PCD cases, indicating a high genetic heterogeneity of this disorder [3]. Mutations in inner dynein arm (IDA) and outer dynein arm (ODA) complex-coding genes cause ciliary abnormalities, but some patients with confirmed PCD clinical features also show normal ciliary motility and ultrastructure [7]. Interestingly, some studies have reported that PCD with heterotaxy and airway ciliary dysfunction only involves 
heterozygous mutations in PCD-associated genes [8]. Another study showed that an autosomal dominant mutation in FOXJ1 can also cause a distinct motile ciliopathy related to defective ciliogenesis, presenting a similar clinical phenotype as that of PCD [9]. These findings suggest the potential involvement of unknown genes and mechanisms in pathologies associated with ciliary function.

Next generation sequencing has advanced the identification of candidate causative genes in PCD [10]. Here, we aimed to identify pathogenic gene variants for sporadic PCD in Chinese patients. Genetic testing combined with TEM and clinical feature analysis have greatly improved the definitive diagnosis of and identification of genetic defects associated with PCD. These tools will allow us to identify risk factors for siblings of individuals with PCD, facilitating the genetic diagnosis of and counseling for this disorder.

\section{Methods}

\section{Subjects and PCD diagnosis}

Five PCD patients, together with control individuals, were enrolled in the study from 2008 to 2020. Patients were diagnosed with PCD according to the classic characterization and guidelines [11]. The control subjects included in the study were the healthy siblings or parents of patients and unrelated healthy individuals. Ethical 
100

101

102

103

104

105

106

107

108

approval was obtained from the ethics committee of the Shenzhen People's Hospital (Guangdong, China), as per the ethical standards recommended in the 1964 Helsinki Declaration of Helsinki. Informed consent was obtained from each subjects enrolled in the study. High-resolution computed tomography (CT), magnetic resonance (MR) imaging, and TEM examination were conducted to investigate chronic sinusitis, bronchiectasis, situs inversus, and ciliary ultrastructural defects.

\section{Identification of variants by whole-exome sequencing (WES) and bioinformatic} analysis

Genomes from all subjects were sequenced using the NovaSeq platform (Illumina). Variants revealed using WES data were analyzed using the Genome Analysis Toolkit and Annovar [12]. Subsequently, all variants were filtered based on minor allele frequency $(\mathrm{MAF}<0.1 \%)$, using the following databases: 1000 genomes, CG69, and gnomAD (http://gnomad.broadinstitute.org/). Sorting Intolerant From Tolerant, MutationTaster, Polymorphism Phenotyping v2 (PolyPhen-2), and Combined Annotation Dependent Depletion were used to assess the pathogenicity of variants. Further, an in-house filtering pipeline was used to identify candidate variants.

\section{Pathogenicity assessment and validation of candidate variants}

Gene function- and frequency-based filtering analyses were conducted for all variants. 
In total, 46 pathogenic genes highly associated with PCD were selected as candidate gene variants, as described previously [13]. Variant pathogenicity assessment was conducted using bioinformatic software and ACMG [14]. The classification and interpretation of variants followed the ACMG guidelines. Identified candidate causative variants were validated in patients with PCD and control subjects using the Sanger sequencing platform. The Chromas software was used to analyze the sequencing results (Technelysium Pty Ltd.).

\section{PCR and sequencing confirmation of the $C C D C 40$ splicing variant}

Total RNA was extracted from whole blood of the PCD patients and reverse-transcribed using the RR036A kit (TAKARA, Japan). Primers for CCDC40 exons were designed using the IDT PrimerQuest tool (https://sg.idtdna.com/Primerquest/Home/Index). cDNA segments transcribed from exon $C C D C 40$ mRNA were amplified using standard PCR. The primers for exon 13 to exon 14 transcripts were as follows: primer 4 F- 5' GGACCAGGACGTGAAGAAAG 3', and R- 5' CTGTGTCACCTTGACCATCTC; and for exon 14 to exon 15 transcripts: primer 6 F- 5' GATCGACGAGCACGATGG 3', and R- GAGCTTCTTCAGGTCGTTGT 3'. Transcript products of the splicing variant in patient KT8 were confirmed by Sanger sequencing and agarose gel electrophoresis. 
143 PCD patients were diagnosed using typical methods, as described in the Materials and

144 Methods section. All patients with PCD had the clinical characteristics of recurrent

145 cough and expectoration for several years (Table 1). Chest CT or MR imaging

146 revealed bronchiectasis in all patients, and situs inversus in four patients (Fig. 1). The

147 four subjects with situs inversus had kartagener syndrome. TEM examination showed

148 the presence of ODA and IDA defects as well as microtubular disorganization of the

149 cilia in KT7 and KT8 (Fig. 1).

150 Table 1. Clinical characteristics of PCD patients

\begin{tabular}{ccccccc}
\hline $\begin{array}{c}\text { PCD } \\
\text { ID }\end{array}$ & Age & Gender & $\begin{array}{c}\text { Recurrent } \\
\text { cough }\end{array}$ & $\begin{array}{c}\text { Situs } \\
\text { inversus }\end{array}$ & Bronchiectasis & Bronchitis \\
\hline KT6 & 30 & Female & Yes & Yes & Yes & Yes \\
KT7 & 32 & Female & Yes & Yes & Yes & Yes \\
KT8 & 48 & Male & Yes & Yes & Yes & Yes \\
KT9 & 48 & Female & Yes & Yes & Yes & No \\
KT10 & 12 & Male & Yes & No & Yes & Yes \\
\hline
\end{tabular}




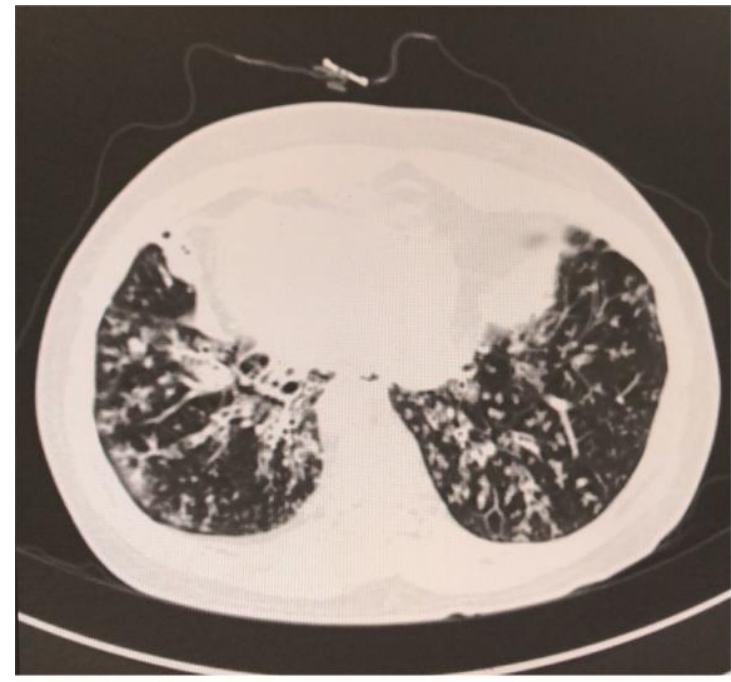

A

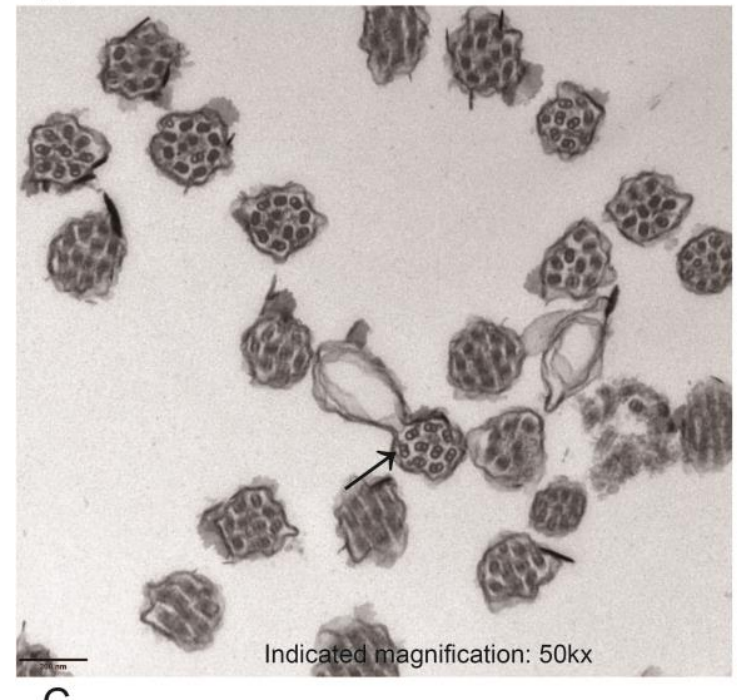

C

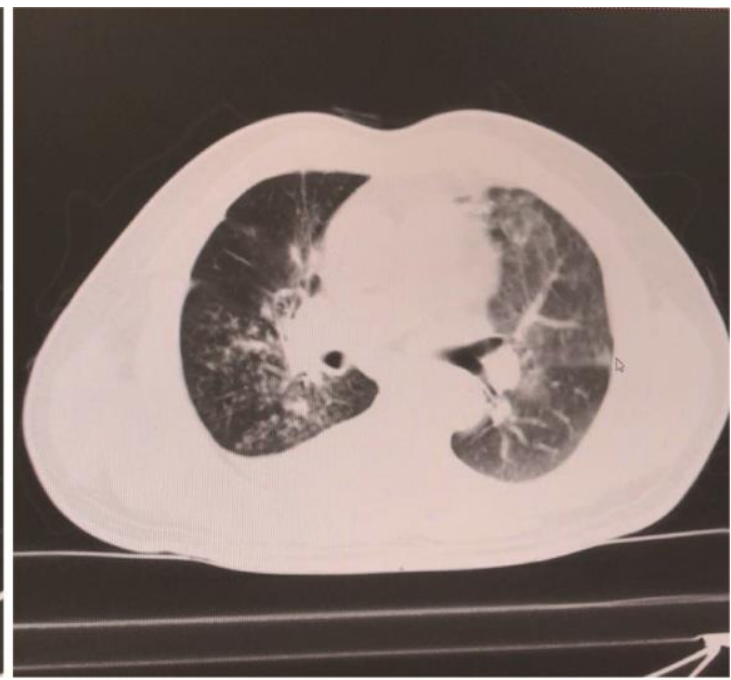

B

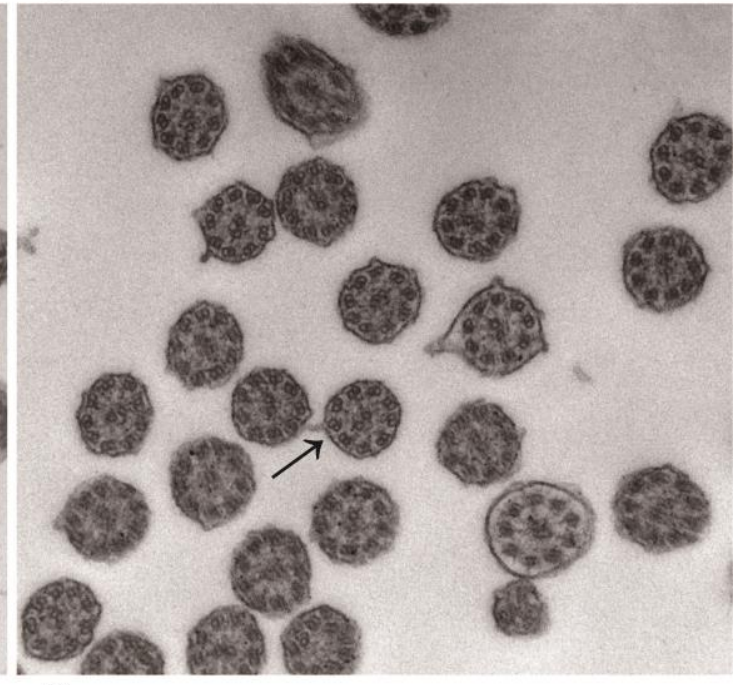

D
153

154

Fig 1. PCD diagnosis using traditional methods. Chest images showed bronchiectasis and situs inversus in KT9 (A) and bronchiectasis in KT10 (B). TEM examination showed structural defects in KT7

(C) and KT8

(D) cilia, the arrows represent the ODA, IDA defects and microtubular disorganization.

\section{Identification of rare variants by bioinformatic analysis}

To identify candidate PCD-causative gene variants in the five Chinese PCD patients, we filtered variants to obtain rare ones based on function and frequency. In total, we 
161

162

163

164

165

166

167

168

169

identified 15 variants of candidate genes with $\mathrm{MAF}<1 \%$ in the East Asian population from the 1000 genomes, ExAC, and gnomAD databases (Table 2). Six novel variants were identified for five genes that were not recorded in the gnomAD and dbSNP databases. We confirmed one homozygous variant of DNAI1 and two homozygous variants of CCDC40 in three patients with PCD to be likely genetic factors contributing to the disorder (Table 2). No other rare heterozygotes were found together with the identified potential pathogenic heterozygous variant in KT6 and KT7, indicating that the genetic defects of these patients were not caused by compound heterozygotes.

Table 2. The interpretation and pathogenicity assessment of candidate variants

\begin{tabular}{|c|c|c|c|c|c|c|c|}
\hline $\begin{array}{c}\text { C } \\
\text { hr }\end{array}$ & Start & $\begin{array}{c}\text { refG } \\
\text { ene }\end{array}$ & Alteration & $\begin{array}{c}\text { PCD } \\
\text { Carrie } \\
\mathbf{r}\end{array}$ & $\begin{array}{c}\text { Varia } \\
\text { nt } \\
\text { type }\end{array}$ & $\begin{array}{c}\text { gno } \\
\text { meA } \\
\text { D } \\
\text { EA } \\
\text { S } \\
\end{array}$ & $\begin{array}{c}\text { ACMG } \\
\text { Classificati } \\
\text { on }\end{array}$ \\
\hline $\begin{array}{l}\mathrm{ch} \\
\mathrm{r} 7\end{array}$ & $\begin{array}{l}2178 \\
1653\end{array}$ & $\begin{array}{l}\text { DNA } \\
\text { H11 }\end{array}$ & $\begin{array}{c}\text { NM_001277115:exon49 } \\
\text { :c.A8023G:p.I2675V }\end{array}$ & KT6 & $\begin{array}{c}\text { Misse } \\
\text { nse }\end{array}$ & $\begin{array}{c}0.000 \\
6\end{array}$ & $\begin{array}{c}\mathrm{PM} 1+\mathrm{PP} 5+ \\
\mathrm{BS} 1+\mathrm{BP} 4\end{array}$ \\
\hline $\begin{array}{c}\mathrm{ch} \\
\mathrm{r} 1 \\
9\end{array}$ & $\begin{array}{l}5567 \\
2134\end{array}$ & $\begin{array}{l}\text { DNA } \\
\text { AF3 }\end{array}$ & $\begin{array}{c}\text { NM_001256714:exon9: } \\
\text { c.G1126T:p.E376X }\end{array}$ & KT7 & $\begin{array}{l}\text { Stop- } \\
\text { gain }\end{array}$ & NA & $\begin{array}{c}\mathrm{PVS} 1+\mathrm{PM} \\
2+\mathrm{PP} 3\end{array}$ \\
\hline $\begin{array}{c}\mathrm{ch} \\
\mathrm{r} 1 \\
7\end{array}$ & $\begin{array}{l}4008 \\
6999\end{array}$ & $\begin{array}{c}\text { TTC } \\
25\end{array}$ & UNKNOWN & KT7 & $\begin{array}{l}\text { Misse } \\
\text { nse }\end{array}$ & NA & $\mathrm{PM} 1+\mathrm{BP} 4$ \\
\hline $\begin{array}{l}\mathrm{ch} \\
\mathrm{r} 3\end{array}$ & $\begin{array}{l}5239 \\
2666\end{array}$ & $\begin{array}{c}\text { DNA } \\
\text { H1 }\end{array}$ & $\begin{array}{c}\text { NM_015512:exon25:c. } \\
\text { C4179A:p.N1393K }\end{array}$ & KT7 & $\begin{array}{l}\text { Misse } \\
\text { nse }\end{array}$ & NA & $\mathrm{PM} 1+\mathrm{BP} 4$ \\
\hline $\begin{array}{l}\mathrm{ch} \\
\mathrm{r} 5\end{array}$ & $\begin{array}{l}1391 \\
1508\end{array}$ & $\begin{array}{c}\text { DNA } \\
\text { H5 }\end{array}$ & $\begin{array}{c}\text { NM_001369:exon12:c. } \\
\text { C1631T:p.T544I }\end{array}$ & KT8 & $\begin{array}{l}\text { Misse } \\
\text { nse }\end{array}$ & $\begin{array}{c}0.009 \\
3\end{array}$ & $\begin{array}{c}\mathrm{PM} 1+\mathrm{PP} 5+ \\
\mathrm{BS} 1+\mathrm{BP} 4\end{array}$ \\
\hline $\begin{array}{l}\mathrm{ch} \\
\mathrm{r} 3\end{array}$ & $\begin{array}{l}5238 \\
8915\end{array}$ & $\begin{array}{c}\text { DNA } \\
\text { H1 }\end{array}$ & $\begin{array}{l}\text { NM_015512:exon21:c. } \\
\text { C3537G:p.Y1179X }\end{array}$ & KT8 & $\begin{array}{l}\text { Stop- } \\
\text { gain }\end{array}$ & NA & $\begin{array}{l}\mathrm{PVS} 1+\mathrm{PM} \\
2+\mathrm{PP} 3\end{array}$ \\
\hline $\begin{array}{l}\mathrm{ch} \\
\mathrm{r} 2\end{array}$ & $\begin{array}{l}8486 \\
1727\end{array}$ & $\begin{array}{c}\text { DNA } \\
\text { H6 }\end{array}$ & $\begin{array}{l}\text { NM_001370:exon30:c. } \\
\text { C4615G:p.Q1539E }\end{array}$ & KT8 & $\begin{array}{l}\text { Misse } \\
\text { nse }\end{array}$ & $\begin{array}{c}0.001 \\
9\end{array}$ & PM1 \\
\hline $\begin{array}{l}\text { chr } \\
14\end{array}$ & $\begin{array}{l}5010 \\
0519\end{array}$ & $\begin{array}{l}\text { DNA } \\
\text { AF2 }\end{array}$ & $\begin{array}{l}\text { NM_001083908:exon1:c. } \\
\text { G1349T:p.S450I }\end{array}$ & KT8 & $\begin{array}{l}\text { Missen } \\
\text { se }\end{array}$ & $\begin{array}{c}0.000 \\
6\end{array}$ & BP4 \\
\hline
\end{tabular}




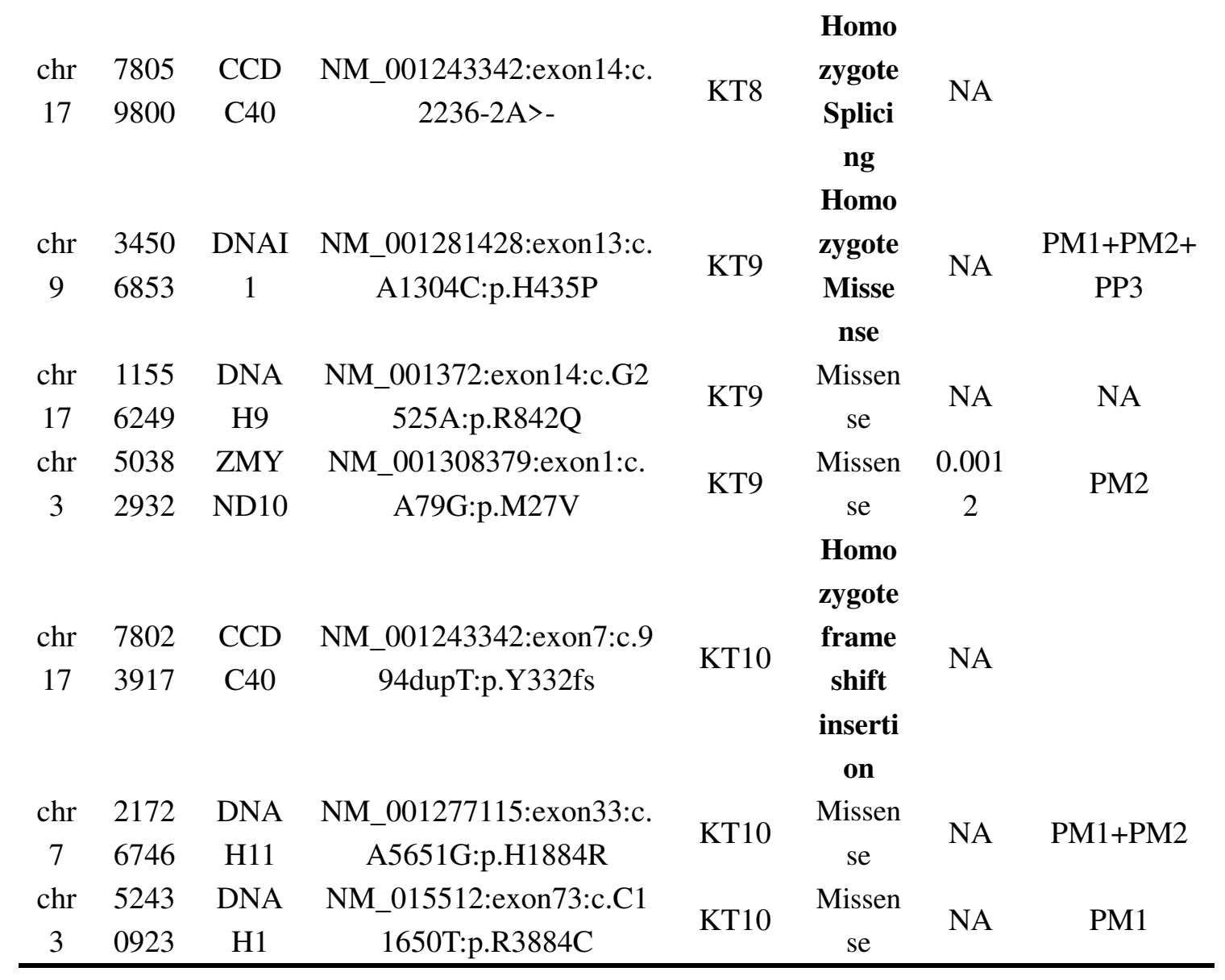

172 ACMG guidelines based pathogenicity assessment of candidate variants

173 Candidate variants were assessed using in silico software and ACMG guidelines

174 (Table 2). All potential causative variants were validated by Sanger sequencing (Fig.

175 2). The frameshift insertion in $C C D C 40$ (c.994dupT) is a pathogenic homozygous

176 variation that was identified as the genetic cause of PCD in KT10. Another

177 homozygous variation in DNAII was present in KT9 in the absence of other rare

178 pathogenic gene variants, the DNAII variant could be the potential cause of PCD,

179 even though it is considered as a VUS according to the ACMG criteria. Further

180 evidence is needed to confirm the association between this homozygous variation in 


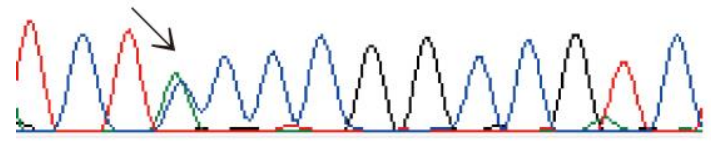

KT7 DNAAF3 c.G1126T
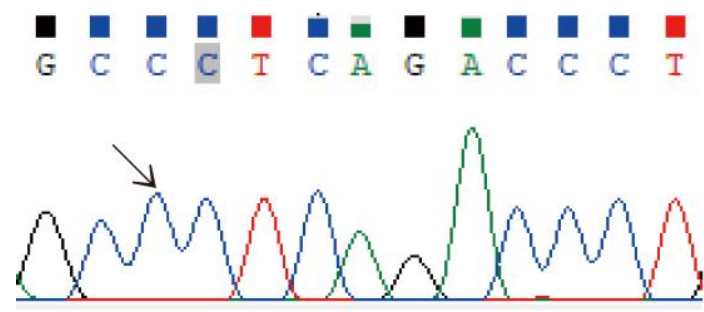
but also by trans-heterozygous gene interactions.

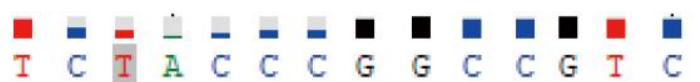

G

$$
\text { KT9 DNAI1 c.A1304C }
$$

185

DNAH1 were identified in KT7 and KT8. We did not find other rare variants of these two genes, indicating that PCD in the two cases was not only caused by heterozygous

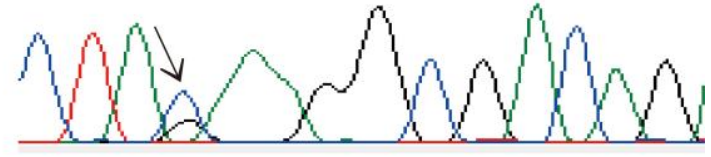

KT8 DNAH1 c.C3537G

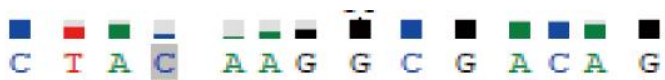

Fig 2. Validation of causative candidate variants by Sanger sequencing.

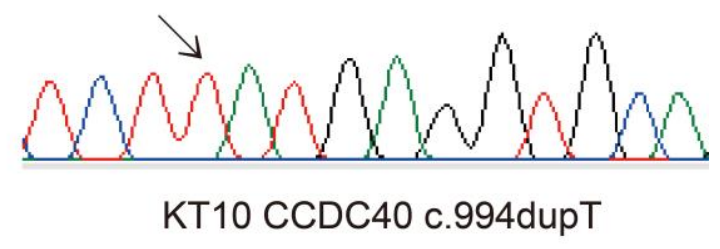

\section{Confirmation of the pathogenicity of the CCDC40 splicing variant}

The homozygous deletion c.2236-2A>- in CCDC40 (NM_017950) found in KT8 alters the nucleotide sequence at the splicing site near exon 14. To confirm whether this variation causes abnormal mRNA transcription, cDNA sequencing was conducted in KT8 and normal control. Genomic DNA sequencing revealed the splicing deletion in KT8 (Fig. 3A). Further PCR and sequencing results showed that the portion of the cDNA between exon 13 and exon 14 was lost in the variant containing the CCDC40 splicing deletion in KT8 (Fig. 3C), while the portion of cDNA was normally present 
in the control. cDNA-based PCR sequencing showed normal CCDC4O cDNA

197 products in the normal control (Fig. 3B). This indicated that the splicing deletion 198 variant of $C C D C 40$ (c.2236-2A>-) resulted in the production of a shorter mRNA 199 transcript compared with normal control. This finding, together with the clinical 200 phenotype resulting from the CCDC4O defect suggest that splicing variant 201 c.2236-2A>- is likely to be pathogenic.
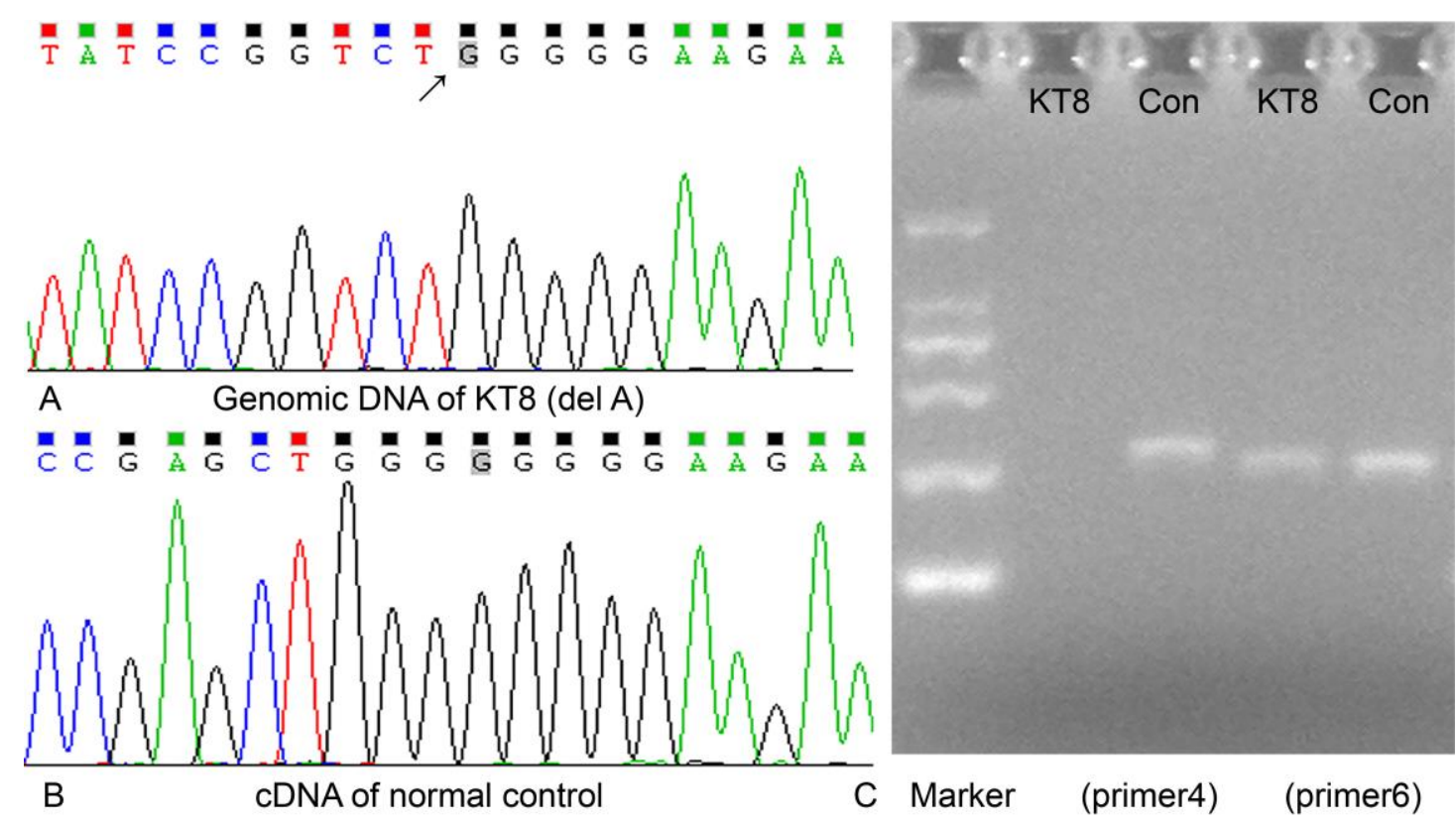

202 Fig 3. (A) Splicing deletion in the KT8 genomic DNA sequence. (B) cDNA 203 sequencing of the control showed a normal sequence between exon 13 and exon 14 of the transcript. (C) Agarose gel electrophoresis showed the CCDC40 cDNA PCR products was lost in KT8.

No rare pathogenic variants were identified in KT6, except for a likely benign rare variant in DNAH1. Two rare variants were predicted as likely benign, and the others 
considered to potentially be causative for PCD based on ACMG guidelines (Table 2).

\section{Discussion}

In the present study, WES was conducted to identify candidate PCD-causative gene variants in five patients. Five causative variations were considered pathogenic in CCDC40, DNAH1, DNAAF3, and DNAI1. Three homozygous variants, including splicing variants, were identified for $C C D C 40$ and DNAI1. Further analyses showed that the splicing variant of $C C D C 40$ might result in the production of a shortened protein, which may contribute to PCD pathogenesis.

To date, there has been strong functional evidence for the contribution of over 50 genes to PCD pathogenesis, with the genes mainly causing structural abnormalities of motile cilia, such as complete or partial deletion of IDAs or ODAs [6]. Defects in showed the classic PCD phenotype involving situs inversus and bronchiectasis. TEM evidence, the splicing deletion variant of $C C D C 40$ may likely be a PCD-causative variant; The exact functions and mechanisms of the splicing variant of CCDC4O in PCD pathogenesis need to be confirmed through further studies. 
One novel PCD-causative homozygous variant was identified in DNAIl. ACMG predicted this variant to be a VUS, but the variant was predicted to be pathogenic by all in silico softwares. DNAII encodes the intermediate chain of ODAs [16]. Mutations in DNAII result in situs inversus and primary ciliary dyskinesia [17]. The carrier of the DNAII variant exhibited the classic PCD phenotype and had no other rare gene variant. We speculated that the DNAII variant is the main genetic factor contributing to PCD in this case. Therefore, appropriate functional studies would be helpful to confirm the pathogenicity of the DNAII variant. We found a novel stop-gain heterozygous variant of DNAAF3 but no other pathogenic variants in one of the assessed PCD patients. The mutation in DNAAF3 causes ODA and IDA defects in cilia [18]. Two rare VUS mutations of DNAH1 and TTC25 were also identified, but whether the observed trans-heterozygous interactions between these mutations could play a role in PCD remains to be confirmed. Identification of ciliary structural defects is helpful to confirm the association between a gene mutation and PCD phenotype. Unfortunately, some patients in our study refused to undergo TEM examination.

As PCD is an autosomal recessive disorder, homozygous or compound heterozygous mutations are commonly considered contributing genetic factors. However, our present and previous studies demonstrated that heterozygous pathogenic mutations are frequent in patients with PCD [13], especially frameshift insertions or deletions and stop-gain or -loss mutations. None of the other candidate pathogenic variants were present together. Interestingly, the inheritance model of the candidate variants is very 
273 inner dynein arm; ODA: outer dynein arm; MAF: minor allele frequency; VUS:

similar to that for autosomal dominant disorders. Some studies have shown that autosomal dominant variants can also cause defects in ciliogenesis with a similar clinical phenotype as that of PCD [9]. However, further investigation is required to determine whether these heterozygous variants can cause PCD on their own.

\section{Conclusion}

In conclusion, our study identified five candidate PCD-causative gene variants of CCDC40, DNAH1, DNAAF3, and DNAI1. More evidence is required to confirm the pathogenicity of various types of genetic variants in PCD, since PCD is a heterogeneous genetic disorders. Confirmation of the causative genetic factors and clinical ciliary defect type in PCD is an important step toward developing personalized clinical diagnosis and genetic counseling.

\section{List of abbreviations}

PCD: Primary ciliary dyskinesia; WES: whole-exome sequencing; TEM: transmission electron microscopy; CT: computed tomography; MR: magnetic resonance; ACMG: the American College of Medical Genetics and Genomics; IDA: variants of uncertain significance. 
277 Shenzhen People's Hospital. All procedures performed in studies involving human participants in accordance with the 1964 Declaration of Helsinki ethical standards.

\section{Consent for publication}

280 Written Informed consent for publication was obtained from all participants, all of the co-author approved the publish of the article.

\section{Availability of data and materials}

The datasets used or analysed during the current study are available from the database of GSA-Human (https://bigd.big.ac.cn/).

\section{Competing interests}

The authors declare that the research was conducted in the absence of any commercial or financial relationships that could be construed as a potential conflict of interest.

\section{$288 \quad$ Funding}

289 The study was supported by the Guangdong Provincial Natural Science Foundation 290 (2018A030310674), the Guangdong Provincial Science and Technology Project 291 (2017A020214016), the Key Laboratory of Shenzhen Respiratory Diseases 292 (ZDSYS201504301616234), and Shenzhen Science and Technology Project 293 (JCYJ20170413093032806). 
295

296

297

298

299

300

301

302

303

304

305

306

307

308

309

310

311

312

313

Yongjian Yue and Yingyun $\mathrm{Fu}$ prepared the project proposal and study design. Yongjian Yue and Lipeng Chen conducted bioinformatics and statistical analysis of sequencing data. Qijun Huang, Fang Yuan, Chunxian Liang, and Kaixue Zhuang conducted sample collection and Sanger sequencing validation. Qijun Huang, Xiangxia Zhang, and Yutian Ye conducted clinical diagnosis of PCD. Tao Liu, Jie Li and Rongchang Chen assisted with the prepared and revised manuscript. All the authors have read and approved the final manuscript.

\section{Acknowledgments}

Thanks to laboratory support from the Key Laboratory of Shenzhen Respiratory Diseases.

\section{Reference}

1. Horani A, Ferkol TW. Advances in the Genetics of Primary Ciliary Dyskinesia: Clinical Implications. Chest. 2018;154(3):645-52.

2. Bush A, Chodhari R, Collins N, Copeland F, Hall P, Harcourt J, et al. Primary ciliary dyskinesia: current state of the art. Arch Dis Child. 2007;92(12):1136-40.

3. Lucas JS, Paff T, Goggin P, Haarman E. Diagnostic Methods in Primary Ciliary Dyskinesia. Paediatric respiratory reviews. 2016;18:8-17.

4. Liu C, Miyata H, Gao Y, Sha Y, Tang S, Xu Z, et al. Bi-allelic DNAH8 Variants 
Lead to Multiple Morphological Abnormalities of the Sperm Flagella and Primary Male Infertility. Am J Hum Genet. 2020;107(2):330-41.

5. Khalid F, Hannah WB, Gaston BM. Rapid Advances in Primary Ciliary Dyskinesia Research. A Brief Update for Pulmonologists. Am J Respir Crit Care Med. 2019;199(2):136-8.

6. Lucas JS, Davis SD, Omran H, Shoemark A. Primary ciliary dyskinesia in the genomics age. Lancet Respir Med. 2020;8(2):202-16.

7. Wallmeier J, Al-Mutairi DA, Chen CT, Loges NT, Pennekamp P, Menchen T, et al. Mutations in CCNO result in congenital mucociliary clearance disorder with reduced generation of multiple motile cilia. Nat Genet. 2014;46(6):646-51.

8. Wilson CW, Nguyen CT, Chen MH, Yang JH, Gacayan R, Huang J, et al. Fused has evolved divergent roles in vertebrate Hedgehog signalling and motile ciliogenesis. Nature. 2009;459(7243):98-102.

9. Wallmeier J, Frank D, Shoemark A, Nothe-Menchen T, Cindric S, Olbrich H, et al. De Novo Mutations in FOXJ1 Result in a Motile Ciliopathy with Hydrocephalus and Randomization of Left/Right Body Asymmetry. Am J Hum Genet. 2019;105(5):1030-9.

10. Fassad MR, Patel MP, Shoemark A, Cullup T, Hayward J, Dixon M, et al. Clinical utility of NGS diagnosis and disease stratification in a multiethnic primary ciliary dyskinesia cohort. J Med Genet. 2020;57(5):322-30.

11. Shapiro AJ, Davis SD, Polineni D, Manion M, Rosenfeld M, Dell SD, et al. Diagnosis of Primary Ciliary Dyskinesia. An Official American Thoracic Society 
Clinical Practice Guideline. Am J Respir Crit Care Med. 2018;197(12):e24-e39.

12. Wang K, Li M, Hakonarson H. ANNOVAR: functional annotation of genetic variants from high-throughput sequencing data. Nucleic Acids Res. 2010;38(16):e164.

13. Yue Y, Huang Q, Zhu P, Zhao P, Tan X, Liu S, et al. Identification of Pathogenic Mutations and Investigation of the NOTCH Pathway Activation in Kartagener Syndrome. Frontiers in genetics. 2019;10:749.

14. Richards S, Aziz N, Bale S, Bick D, Das S, Gastier-Foster J, et al. Standards and guidelines for the interpretation of sequence variants: a joint consensus recommendation of the American College of Medical Genetics and Genomics and the Association for Molecular Pathology. Genet Med. 2015;17(5):405-24.

15. Becker-Heck A, Zohn IE, Okabe N, Pollock A, Lenhart KB, Sullivan-Brown J, et al. The coiled-coil domain containing protein CCDC40 is essential for motile cilia function and left-right axis formation. Nat Genet. 2011;43(1):79-84.

16. Zariwala MA, Leigh MW, Ceppa F, Kennedy MP, Noone PG, Carson JL, et al. Mutations of DNAI1 in primary ciliary dyskinesia: evidence of founder effect in a common mutation. Am J Respir Crit Care Med. 2006;174(8):858-66.

17. Guichard C, Harricane MC, Lafitte JJ, Godard P, Zaegel M, Tack V, et al. Axonemal dynein intermediate-chain gene (DNAI1) mutations result in situs inversus and primary ciliary dyskinesia (Kartagener syndrome). Am J Hum Genet. 2001;68(4):1030-5.

18. Mitchison HM, Schmidts M, Loges NT, Freshour J, Dritsoula A, Hirst RA, et al. Mutations in axonemal dynein assembly factor DNAAF3 cause primary ciliary 


\section{Figure legends}

360 Fig 1. PCD diagnosis using traditional methods. Chest images showed bronchiectasis 361 and situs inversus in KT9 (A) and bronchiectasis in KT10 (B). TEM examination 362 showed structural defects in KT7 (C) and KT8 (D) cilia, the arrows represent the 363 ODA, IDA defects and microtubular disorganization.

364

Fig 2. Validation of causative candidate variants by Sanger sequencing.

Fig 3. (A) Splicing deletion in the KT8 genomic DNA sequence. (B) cDNA 368 sequencing of the control showed a normal sequence between exon 13 and exon 14 of products was lost in KT8. 


\section{Figures}
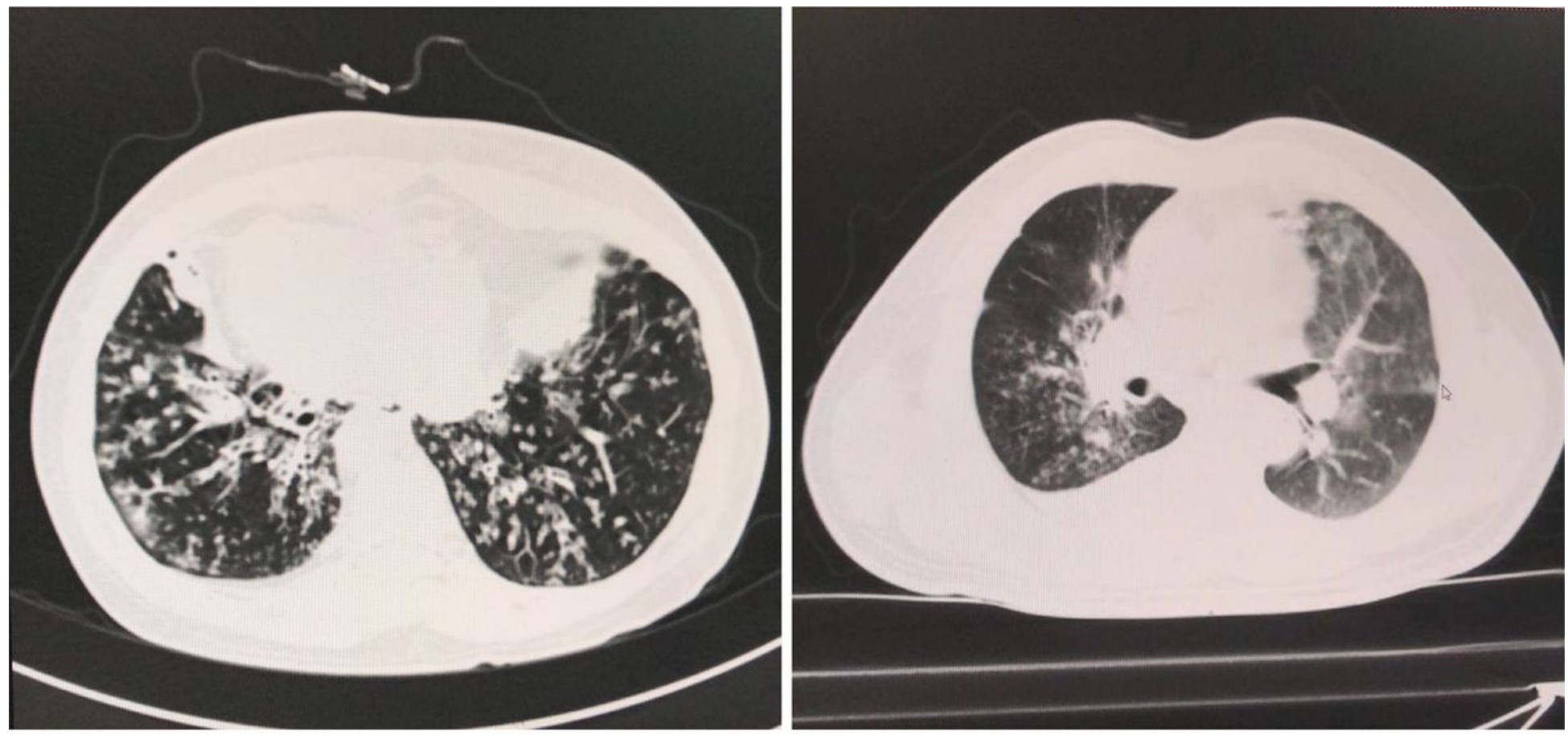

A

B
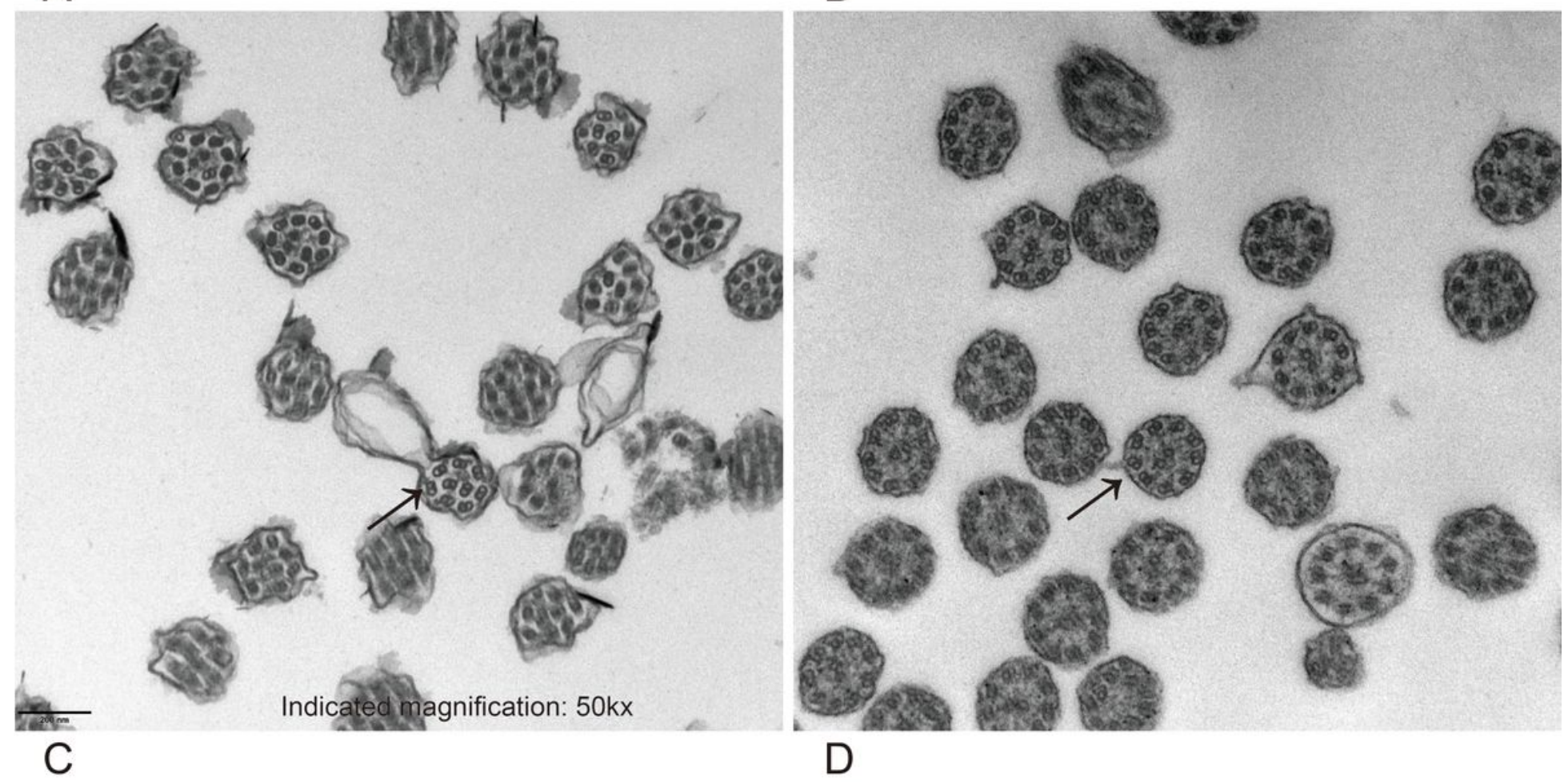

Figure 1

PCD diagnosis using traditional methods. Chest images showed bronchiectasis and situs inversus in KT9 (A) and bronchiectasis in KT10 (B). TEM examination showed structural defects in KT7 (C) and KT8 (D) cilia, the arrows represent the ODA, IDA defects and microtubular disorganization. 
I C I I A C C C G G

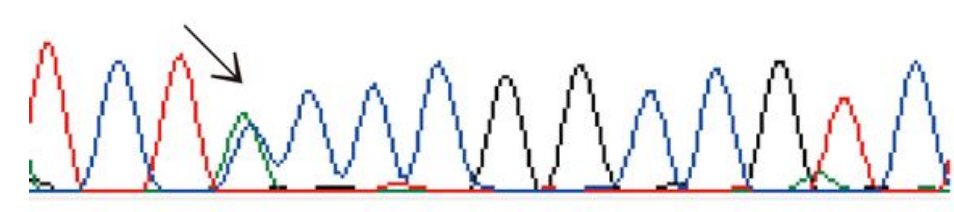

KT7 DNAAF3 c.G1126T

घ

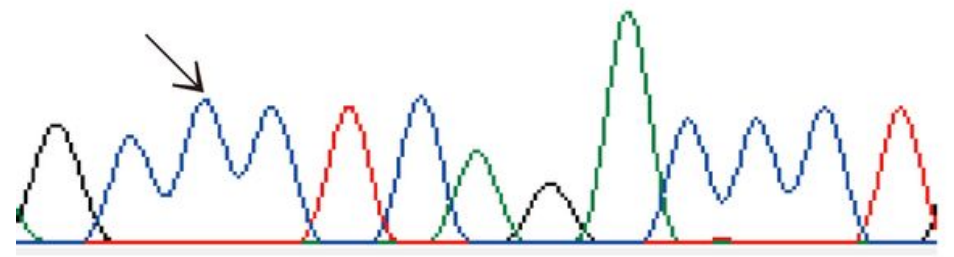

KT9 DNAI1 c.A1304C

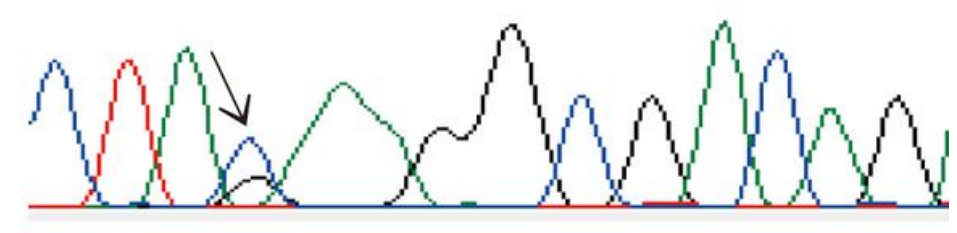

KT8 DNAH1 c.C3537G

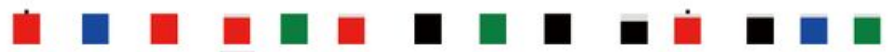

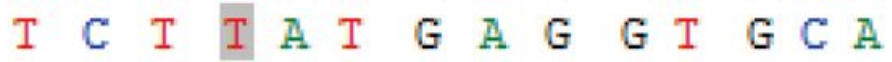

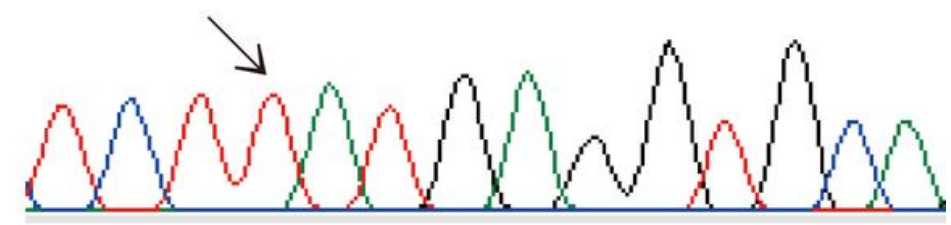

KT10 CCDC40 c.994dupT

Figure 2

Validation of causative candidate variants by Sanger sequencing.

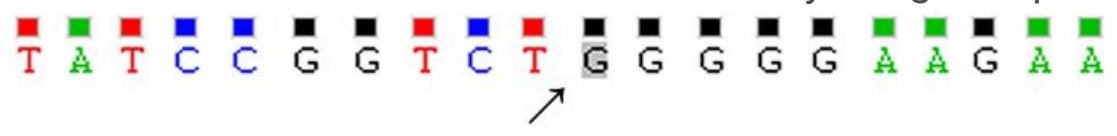
KT8 Con KT8 Con

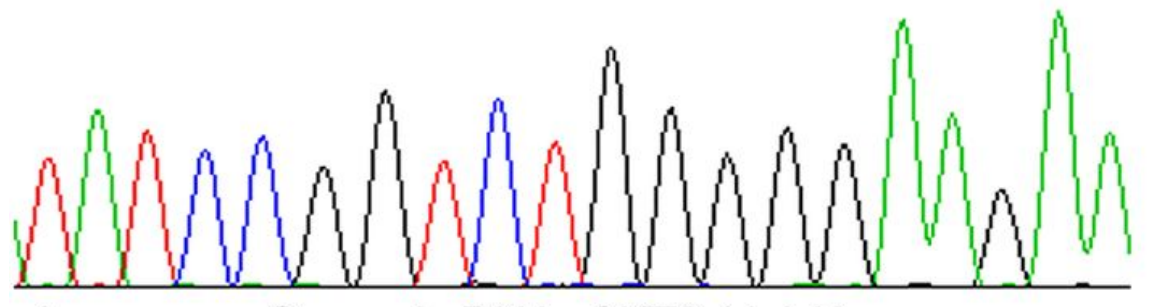

A Genomic DNA of KT8 (del A)

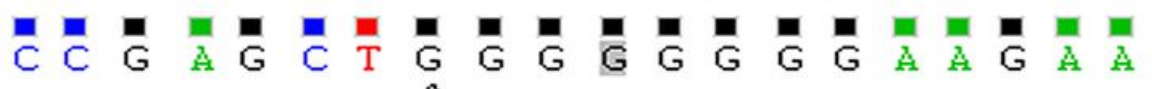

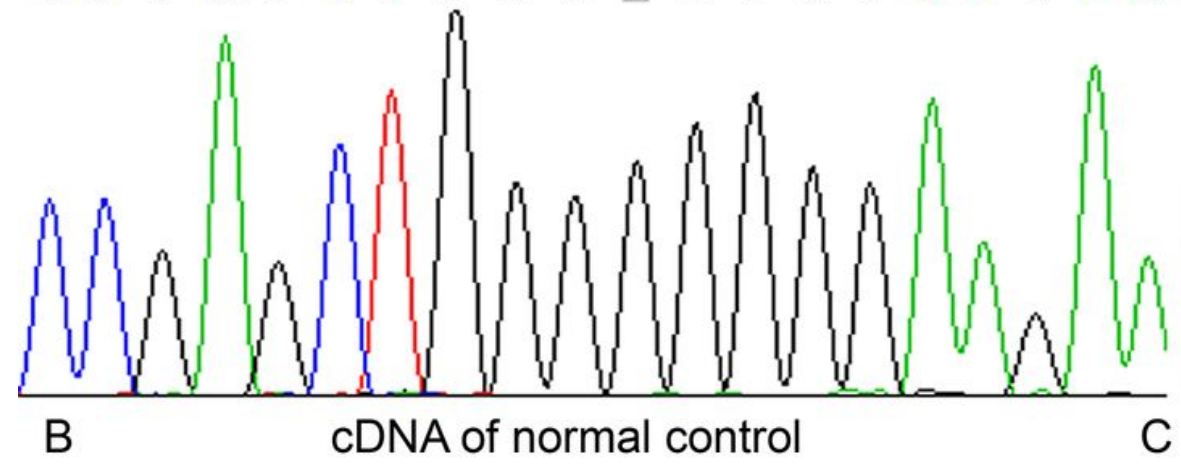

C Marker

(primer4)

(primer6) 
Figure 3

(A) Splicing deletion in the KT8 genomic DNA sequence. (B) cDNA sequencing of the control showed a normal sequence between exon 13 and exon 14 of the transcript. (C) Agarose gel electrophoresis showed the CCDC40 cDNA PCR products was lost in KT8. 